\title{
Therapeutic drug monitoring using saliva as matrix: an opportunity for linezolid, but challenge for moxifloxacin
}

\author{
To the Editor:
}

The World Health Organization (WHO) has listed moxifloxacin and linezolid among the preferred "group A" drugs in the treatment of multidrug-resistant (MDR)-tuberculosis (TB) [1]. Therapeutic drug monitoring (TDM) could potentially optimise MDR-TB therapy, since moxifloxacin and linezolid show large pharmacokinetic variability [1-4]. TDM of moxifloxacin focuses on identifying patients with low drug exposure who are at risk of treatment failure and acquired fluoroquinolone resistance [5, 6]. Alternatively, TDM of linezolid strives to reduce toxicity while ensuring an adequate drug exposure because of its narrow therapeutic index $[1,3,7]$.

TDM is typically performed using plasma or serum samples, but other biological matrices can be considered as alternatives (e.g. saliva) [8]. A benefit of saliva is the easy and non-invasive nature of sampling. Especially in high TB burden areas, the option for sampling at home would be advantageous. Penetration of moxifloxacin into saliva has typically been studied in healthy volunteers, but has never been evaluated for the purpose of TDM in MDR-TB patients [9]. Only one study described linezolid concentrations in saliva and found that saliva is a suitable matrix for TDM in MDR-TB patients and that salivary concentrations can be translated to serum concentrations without the need of a correction factor [7]. The aim of this prospective study was to explore the feasibility of saliva-based TDM of moxifloxacin and to determine if earlier results of linezolid in saliva of MDR-TB patients could be confirmed.

Hospitalised adult TB patients in the Tuberculosis Center Beatrixoord (Haren, the Netherlands), who had moxifloxacin or linezolid as part of their TB treatment and had routine TDM using blood samples were eligible for inclusion. All participants signed informed consent. This study was registered at Clinicaltrials. gov (NCT03080012) and approved by the ethical review committee of the University Medical Center Groningen (IRB 2016/069).

After at least 14 days of treatment, saliva samples were taken simultaneously with plasma (moxifloxacin) or serum (linezolid) according to routine TDM schedule which generally included a sample before and $1,2,3,4$ and $8 \mathrm{~h}$ after drug administration. All samples were stored at $-80 \mathrm{C}$ until analysis.

To collect saliva samples, patients were asked to chew on a cotton roll after rinsing their mouth with water. Subsequently, the samples were processed using one of the following methods. Salivette (Sarstedt, Nümbrecht, Germany) in combination with centrifugation was used for non-contagious patients. Membrane filtration was applied to the saliva samples of the TB patients who still had Mycobacterium tuberculosis bacilli in their sputum to minimise infection hazard [10]. Salivary pH values were determined by two independent observers using $\mathrm{pH}$ indicator strips with $\mathrm{pH}$ range 4.0-7.0 and 2.0-9.0 (Merck KGaA, Darmstadt, Germany), because it could influence drug penetration into saliva [9]. Recovery of both sampling methods was determined similarly to the method reported by GHImIRE et al. [11], except that moxifloxacin was tested at 1 and $3 \mathrm{mg} \cdot \mathrm{L}^{-1}$ and linezolid at 2 and $20 \mathrm{mg} \cdot \mathrm{L}^{-1}$. Recovery was comparable for low and high concentrations. Using the Salivette, recovery was $48 \%$ (coefficient of variation (CV) $6 \%$ ) for moxifloxacin or 95\% (CV 3\%) for linezolid, and via membrane filtration 48\% (CV 6\%) or 98\% (CV 3\%), respectively. After analysis the salivary concentrations were corrected for recovery accordingly.

@ERSpublications

Therapeutic drug monitoring using saliva as matrix is a suitable alternative for serum therapeutic drug monitoring of linezolid, but not for moxifloxacin due to a high variability in saliva-plasma ratios http://bit.ly/2NIYdz7

Cite this article as: van den Elsen SHJ, Akkerman OW, Jongedijk EM, et al. Therapeutic drug monitoring using saliva as matrix: an opportunity for linezolid, but challenge for moxifloxacin. Eur Respir J 2020; 55 : 1901903 [https://doi.org/10.1183/13993003.01903-2019]. 
All samples were analysed using an updated version of our previously published liquid chromatography tandem mass spectrometry (LC-MS/MS) method [12, 13]. The LC-MS/MS method of linezolid was already cross-validated for saliva [7]. Cross-validation between plasma and saliva was performed for moxifloxacin at low $\left(1 \mathrm{mg} \cdot \mathrm{L}^{-1}\right)$, medium $\left(2 \mathrm{mg} \cdot \mathrm{L}^{-1}\right)$, and high $\left(4 \mathrm{mg} \cdot \mathrm{L}^{-1}\right)$ concentrations as well as at lower limit of quantification (LLOQ; $0.05 \mathrm{mg} \cdot \mathrm{L}^{-1}$ ). All concentration levels met the pre-set criteria for accuracy and precision (bias and CV $<15 \%$; at LLOQ both $<20 \%$ ).

Area under the concentration-time curve from 0 to $24 \mathrm{~h}\left(\mathrm{AUC}_{0-24}\right)$ in saliva and plasma/serum was calculated using noncompartmental pharmacokinetic analysis (MWPharm version 3.82, Mediware, Groningen, the Netherlands). $\mathrm{C}_{\max }$ was defined as highest observed concentration and $\mathrm{T}_{\max }$ as corresponding time of $\mathrm{C}_{\max }$. Two different saliva to plasma/serum ratios were calculated; one used the paired drug concentrations, while the other compared $\mathrm{AUC}_{0-24}$ in both matrices. Passing-Bablok regression and Bland-Altman plots (Analyze-it 4.81; Analyze-it Software Ltd, Leeds, UK) were used to analyse results.

Patient characteristics, pharmacokinetic parameters $\left(C_{\max }, T_{\max }, A C_{0-24}\right)$, and saliva to serum/plasma ratios are shown in table 1 . All patients on linezolid also received moxifloxacin. Individual linezolid concentration-time curves in saliva versus serum were similarly shaped and $\mathrm{T}_{\max }$ in saliva was not delayed, which suggested that penetration of linezolid into saliva is fast. Passing-Bablok analysis showed a linear regression line of saliva concentration $=0.389+0.680 \times$ serum concentration with $95 \%$ CI of intercept -0.14 to 1.06 ; $95 \%$ CI of slope 0.60 to 0.76 ; $r=0.954$, and $\mathrm{p}=0.519$. Bland-Altman demonstrated a mean saliva to serum concentration ratio of 0.76 (95\% CI $0.70-0.82)$. In general, the linezolid saliva to serum paired concentration ratio was generally constant at $0.6-0.8$ (range $0.25-1.29$ ). Saliva to serum $\mathrm{AUC}_{0-24}$ ratios were even less variable, with a median of 0.81 (range $0.54-0.96$ ). However, we found a lower saliva to serum ratio than before [7], which could be caused by differences in sampling method, processing or storage. Because linezolid efficacy is related to the ratio of $\mathrm{AUC}_{0-24}$ to minimal inhibitory concentration $\left(\mathrm{AUC}_{0-24} / \mathrm{MIC}\right)$, it is recommended to collect multiple saliva samples to calculate $\mathrm{AUC}_{0-24}$ in saliva and afterwards translate to plasma $\mathrm{AUC}_{0-24}$ using a correction factor of 1.2. Based on these results, salivary TDM of linezolid indeed might be feasible and is ready for testing in a high burden TB setting. Although LC-MS/MS was used in our study, high-performance liquid chromatography with UV detection could be a suitable alternative in less resourced settings. Simple point-of-care tests in saliva, centralised drug analysis, stability studies for transport at room temperature conditions, and cross-validation of existing analytical methods in saliva may improve feasibility [8].

Moxifloxacin paired saliva to plasma concentration ratios were highly variable with a range of $0.15-2.81$ (median 1.00), which does not favour saliva as a sampling matrix for TDM. Passing-Bablok showed a

TABLE 1 Characteristics of the linezolid and moxifloxacin study populations, pharmacokinetic (PK) parameters in serum/plasma and saliva, and saliva to serum/plasma ratios using paired concentrations as well as area under the concentration-time curve from 0 to $24 \mathrm{~h}\left(\mathrm{AUC}_{0-24}\right)$

\begin{tabular}{|c|c|c|}
\hline & Linezolid (n=7) & Moxifloxacin (n=15) \\
\hline \multicolumn{3}{|l|}{ Study population } \\
\hline Male & $6(86 \%)$ & $11(73 \%)$ \\
\hline Age years & $44(37-55)$ & $34(25-55)$ \\
\hline Bodyweight kg & $67.1(60.5-68.4)$ & $67.1(57.5-70.5)$ \\
\hline Creatinine concentration $\mu \mathrm{mol} \cdot \mathrm{L}^{-1}$ & $73(72-90)$ & (72 (63-90) \\
\hline Dose $\mathrm{mg} \cdot \mathrm{kg}^{-1}$ & $8.85(7.42-9.93)$ & $5.96(5.68-7.08)$ \\
\hline \multicolumn{3}{|l|}{ Serum/plasma $\mathrm{PK}^{\#}$} \\
\hline $\mathrm{C}_{\max } \mathrm{mg} \cdot \mathrm{L}^{-1}$ & $12.45(8.84-15.78)$ & $2.28(1.62-2.80)$ \\
\hline$T_{\max } h$ & $3(2-4)$ & $2(1-2)$ \\
\hline $\mathrm{AUC}_{0-24} \mathrm{mg} \cdot \mathrm{h} \cdot \mathrm{L}^{-1}$ & $119.4(116.2-128.2)$ & $21.3(15.8-31.0)$ \\
\hline \multicolumn{3}{|l|}{ Saliva PK } \\
\hline $\mathrm{C}_{\max } \mathrm{mg} \cdot \mathrm{L}^{-1}$ & $7.93(7.55-12.38)$ & $3.20(2.51-4.25)$ \\
\hline $\mathrm{T}_{\max }(\mathrm{h})$ & $3(2-3)$ & $2(1-2)$ \\
\hline $\mathrm{AUC}_{0-24} \mathrm{mg} \cdot \mathrm{h} \cdot \mathrm{L}^{-1}$ & $93.6(91.7-108.0)$ & $21.3(13.7-28.3)$ \\
\hline \multicolumn{3}{|l|}{ Saliva to serum/plasma ratio" } \\
\hline Paired concentration ratio & $0.76(0.64-0.85)$ & $1.00(0.68-1.35)$ \\
\hline $\mathrm{AUC}_{0-24}$ ratio & $0.81(0.74-0.88)$ & $0.89(0.61-1.14)$ \\
\hline
\end{tabular}


linear relation of saliva concentration $=-0.620+1.49 \times$ serum concentration, $95 \%$ CI intercept -0.97 to -0.33 , $95 \%$ CI slope 1.32 to $1.74 ; \mathrm{r}=0.796$, and $\mathrm{p}=0.103$. As moxifloxacin saliva to plasma concentration ratios were not normally distributed according to Shapiro-Wilk test $(\mathrm{p}=0.0003)$, Bland-Altman analysis could not be used. Unfortunately, saliva-plasma $\mathrm{AUC}_{0-24}$ ratios showed similar results (range 0.30-2.00), but the underlying cause remains unclear. Both inter-individual as well as intra-individual variation was observed. No effect of salivary $\mathrm{pH}$ on saliva to plasma ratios of moxifloxacin could be detected. A limitation of our study was that we did not measure the unbound concentrations. Therefore, variation in protein binding could have affected the saliva to plasma ratios. Interestingly, salivary concentrations higher than plasma concentrations were observed, suggesting possibilities of active transport in addition to passive diffusion [9]. Moxifloxacin also shows excellent penetration into diseased lung tissue with a median free-tissue to free-serum ratio of 3.2 [14]. It would be interesting to investigate whether salivary concentrations are related to tissue concentrations at the site of infection and if penetration is driven by similar mechanisms. If closely related, it might be possible to determine infection site moxifloxacin concentrations without the need of invasive tissue sampling. Clearly, it is the free drug concentrations at the site of action that is predictive of treatment efficacy, while plasma moxifloxacin concentrations serve as no more than proxy markers.

Salivary TDM could be an alternative method for traditional linezolid TDM using plasma or serum, and future studies can focus on improving the feasibility. However, for moxifloxacin our data does not support saliva as suitable matrix for TDM using the described method. Future studies should investigate moxifloxacin protein binding, salivary flow and transport mechanisms to gain more insight in the feasibility of moxifloxacin TDM in saliva. As shown before for amikacin [15], saliva will likely not be a universal but only a selective matrix for TDM of anti-TB drugs.

Simone H.J. van den Elsen ${ }^{1}$, Onno W. Akkerman ${ }^{2,3}$, Erwin M. Jongedijk ${ }^{1}$, Mireille Wessels ${ }^{1}$, Samiksha Ghimire ${ }^{1}$, Tjip S. van der Werf ${ }^{3,4}$, Daan J. Touw ${ }^{1}$, Mathieu S. Bolhuis ${ }^{1}$ and Jan-Willem C. Alffenaar ${ }^{1,5,6,7}$

${ }^{1}$ University of Groningen, University Medical Center Groningen, Dept of Clinical Pharmacy and Pharmacology, Groningen, The Netherlands. ${ }^{2}$ University of Groningen, University Medical Center Groningen, Tuberculosis Center Beatrixoord, Haren, The Netherlands. ${ }^{3}$ University of Groningen, University Medical Center Groningen, Dept of Pulmonary Diseases and Tuberculosis, Groningen, The Netherlands. ${ }^{4}$ University of Groningen, University Medical Center Groningen, Dept of Internal Medicine, Groningen, The Netherlands. ${ }^{5}$ Sydney Pharmacy School, Faculty of Medicine and Health, The University of Sydney, Sydney, Australia. ${ }^{6}$ Westmead hospital, Sydney, Australia. ${ }^{7}$ Marie Bashir Institute of Infectious Diseases and Biosecurity, Sydney, Australia

Correspondence: Jan-Willem C. Alffenaar, University of Sydney, Faculty of Medicine and Health, School of Pharmacy, Pharmacy Building A15, NSW 2006, Australia. E-mail: johannes.alffenaar@sydney.edu.au

Received: 25 Sept 2019 | Accepted after revision: 10 Jan 2020

This study was registered at Clinicaltrials.gov with identifier number NCT03080012.

Conflict of interest: None declared.

\section{References}

1 World Health Organization. WHO Consolidated Guidelines on Drug-resistant Tuberculosis Treatment. Geneva, World Health Organization, 2019.

2 Pranger $\mathrm{AD}$, van Altena R, Aarnoutse RE, et al. Evaluation of moxifloxacin for the treatment of tuberculosis: 3 years of experience. Eur Respir J 2011; 38: 888-894.

3 Bolhuis MS, Akkerman OW, Sturkenboom MGG, et al. Linezolid-based regimens for multidrug-resistant tuberculosis (TB): a systematic review to establish or revise the current recommended dose for TB treatment. Clin Infect Dis 2018; 67: S327-S335.

4 Srivastava S, Peloquin CA, Sotgiu G, et al. Therapeutic drug management: is it the future of multidrug-resistant tuberculosis treatment? Eur Respir J 2013; 42: 1449-1453.

5 Davies Forsman L, Bruchfeld J, Alffenaar J-WC. Therapeutic drug monitoring to prevent acquired drug resistance of fluoroquinolones in the treatment of tuberculosis. Eur Respir J 2017; 49: 1700173.

6 Pranger AD, van der Werf TS, Kosterink JGW, et al. The role of fluoroquinolones in the treatment of tuberculosis in 2019. Drugs 2019; 79: 161-171.

7 Bolhuis MS, van Altena R, van Hateren K, et al. Clinical validation of the analysis of linezolid and clarithromycin in oral fluid of patients with multidrug-resistant tuberculosis. Antimicrob Agents Chemother 2013; 57: 3676-3680.

8 Ghimire S, Bolhuis MS, Sturkenboom MGG, et al. Incorporating therapeutic drug monitoring into the World Health Organization hierarchy of tuberculosis diagnostics. Eur Respir J 2016; 47: 1867-1869.

9 van den Elsen SHJ, Oostenbrink LM, Heysell SK, et al. Systematic review of salivary versus blood concentrations of antituberculosis drugs and their potential for salivary therapeutic drug monitoring. Ther Drug Monit 2018; 40: $17-37$.

10 van den Elsen SHJ, van der Laan T, Akkerman OW, et al. Membrane filtration is suitable for reliable elimination of Mycobacterium tuberculosis from saliva for therapeutic drug monitoring. J Clin Microbiol 2017; 55: 3292-3293.

11 Ghimire S, Maharjan B, Jongedijk EM, et al. Evaluation of saliva as a potential alternative sampling matrix for therapeutic drug monitoring of levofloxacin in patients with multidrug-resistant tuberculosis. Antimicrob Agents Chemother 2019; 63: e02379-18. 
12 Harmelink IM, Alffenaar JW, Wessels M, et al. A rapid and simple liquid chromatography-tandem mass spectrometry method for the determination of linezolid in human serum. EJHP Sci 2008; 14: 3-7.

13 Pranger AD, Alffenaar J-WC, Wessels AMA, et al. Determination of moxifloxacin in human plasma, plasma ultrafiltrate, and cerebrospinal fluid by a rapid and simple liquid chromatography- tandem mass spectrometry method. J Anal Toxicol 2010; 34: 135-141.

14 Heinrichs MT, Vashakidze S, Nikolaishvili K, et al. Moxifloxacin target site concentrations in patients with pulmonary TB utilizing microdialysis: a clinical pharmacokinetic study. J Antimicrob Chemother 2018; 73: $477-483$.

15 van den Elsen SHJ, Akkerman OW, Huisman JR, et al. Lack of penetration of amikacin into saliva of tuberculosis patients. Eur Respir J 2018; 51: 1702024.

Copyright (CERS 2020 